\title{
Real-time Hybrid Locomotion Mode Recognition for Lower-limb Wearable Robots
}

\author{
Andrea Parri ${ }^{\dagger}$, Kebin Yuan ${ }^{\dagger}$, Student Member, IEEE, Dario Marconi, Tingfang Yan, Simona Crea, Member, IEEE, \\ Marko Munih, Member, IEEE, Raffaele Molino Lova, \\ Nicola Vitiello*, Member, IEEE, and Qining Wang*, Member, IEEE
}

\begin{abstract}
Real-time recognition of locomotion-related activities is a fundamental skill that a controller of lower-limb wearable robots should possess. Subject-specific training and reliance on electromyographic interfaces are the main limitations of existing approaches. This study presents a novel methodology for realtime locomotion mode recognition of locomotion-related activities in lower-limb wearable robotics. A hybrid classifier can distinguish among seven locomotion-related activities. First, a timebased approach classifies between static and dynamical states based on gait kinematics data. Second, an event-based fuzzy logic method triggered by foot pressure sensors operates in a subjectindependent fashion on a minimal set of relevant biomechanical features to classify among dynamical modes. The locomotion mode recognition algorithm is implemented on the controller of a portable powered orthosis for hip assistance. An experimental protocol is designed to evaluate the controller performance in an out-of-lab scenario without the need for a subject-specific training. Experiments are conducted on six healthy volunteers performing locomotion-related activities at slow, normal, and fast speeds under the zero-torque and assistive mode of the orthosis. The overall accuracy rate of the controller is $99.4 \%$ over more than 10,000 steps, including seamless transitions between different modes. The experimental results show a successful subject-independent performance of the controller for wearable robots assisting locomotion-related activities.
\end{abstract}

Index Terms-Locomotion mode recognition, fuzzy-logic classifier, lower-limb wearable robots, gait assistance

This work was supported by the EU within the CYBERLEGs project (FP7-ICT-2011-2.1 Grant Agreement \#287894) and CYBERLEGs Plus Plus project (H2020-ICT-2016-1 Grant Agreement \#731931), Fondazione Pisa within the IUVO project (prog. 154/11), the Beijing Disabled Persons' Federation, the Beijing Nova Program (no. Z141101001814001), and the Beijing Municipal Science and Technology Project (nos. Z151100003715001 nad Z151100000915073).

Andrea Parri, Dario Marconi, Simona Crea and Nicola Vitiello are with The BioRobotics Institute, Scuola Superiore Sant'Anna, Italy. (e-mail: [andrea1.parri, dario.marconi, simona.crea, nicola.vitiello]@ santannapisa.it)

Kebin Yuan and Qining Wang are with the Robotics Research Group, College of Engineering, Peking University, Beijing, China. (e-mail: [kebin.yuan, qiningwang]@pku.edu.cn)

Tingfang Yan was with The BioRobotics Institute, Scuola Superiore Sant'Anna. She is now with The Chinese University of Hong Kong. (e-mail: tfyan@ee.cuhk.edu.hk).

Marko Munih is with the Laboratory of Robotics at the Faculty of Electrical Engineering, University of Ljubljana, Slovenia. (e-mail: marko.munih@robo.fe.uni-lj.si).

Raffaele Molino Lova and Nicola Vitiello are with Don Carlo Gnocchi Foundation, Italy. (e-mail: rmolino@dongnocchi.it)

${ }^{\dagger}$ Andrea Parri and Kebin Yuan are equal contributors. ${ }^{*}$ Qining Wang and Nicola Vitiello are equally contributors. Qining Wang is the corresponding author.

Andrea Parri, Simona Crea and Nicola Vitiello have commercial interests in IUVO S.r.l, a spin off company of Scuola Superiore Sant'Anna. Currently, the IP protecting the APO technology has been licensed to IUVO S.r.l. for commercial exploitation.

\section{INTRODUCTION}

$\mathbf{P}$ RESERVING the mobility of lower-limb impaired individuals is a challenging emergency for sustaining the global welfare of our aging society [1]. In this framework, lower-limb assistive wearable robots are a newborn class of technologies intended to promote the recovery and improvement of the locomotor functionality [2], [3]. Given its close cooperation with the human user, by nature adaptive and inherently exhibiting inter- and intra-subject variabilities, a lower limb wearable robot should possess cognitive skills in its control framework [2], [3]. In order to be effective in dailylife activities, a skilled controller should be able to on-line (i) decode the intended movement of the user (e.g., walking, climbing stairs, sitting, or standing up) and (ii) estimate the phase of the intrinsically cyclical locomotion-related tasks adapting to the minor changes caused by intra-cycle variability [4].

Several studies proposed different approaches for the on-line recognition of locomotion-related activities. Recent reviews [4], [5] presented a detailed overview of the sensory networks constituting a human robot interface and of the methods of task recognition. As regards active prostheses, the interfaces for intention decoding based on surface electromyography (sEMG) were the most common [6]-[8]. Sensing based on sEMG was then coupled with the measurements acquired from mechanical sensors such as angular sensors, inertial measurement units (IMUs), or load cells, to embed sensory systems devoted to intention decoding in the prosthetic segments of the mechatronic device itself [9]-[11]. The success rate using sEMG-based interfaces can increase from 93\% [7] up to 97.7\% [10] and 98.9\% [11] when the sEMG and mechanical sensors are combined. The accuracy rate superior to $99 \%$ can be achieved with EMG-based interfaces fostering targeted muscle reinnervation [12]. Nevertheless, the robustness of EMG sensing is prone to fade in prolonged use because of skin temperature variations, sweating, and relative movements between the skin and electrodes. Therefore, alternative approaches have more recently abandoned sEMG electrodes preferring networks of mechanical sensors to minimize the invasiveness of the interface and increase its dependability [13]-[16].

The success rate of decoding methods depends not only on the sensory system constituting the interface, but also on the techniques used for classification. For studies based on mechanical sensing, simple threshold-based finite state machine controllers provided an accuracy rate not higher than 
$97 \%$ [13], [14]. The success rate improved up to $98.8 \%$ when processing (e.g., using fuzzy logic-based classifiers) [15] or linear discriminant analysis [16] techniques are applied. A subject-specific training is often unavoidable for these approaches, and large training pools of data are needed to properly define the transitioning rules.

Several algorithms for the locomotion mode recognition (LMR) were also developed for powered lower-limb exoskeletons [17]. As regards assisting ground-level walking, variable stiffness actuation is a common adopted strategy for adapting the stiffness of actuated joints depending on the walking terrain [18]-[20]. A successful approach for the LMR of patients with no residual neuromuscular peripheral activities (e.g., patients with spinal cord injury) is represented by brain machine interfaces (BMIs) for triggering gait initiation and termination and steady walking tasks [21]-[23]. Nevertheless, the BMI complexity limits their application in daily-life ambulation. Therefore, commercial lower-limb orthoses for patients with spinal cord injury are often controlled monitoring mechanical feature of the human-exoskeleton system, such as the tilting of the trunk [24], [25]. The most advanced approach for the LMR with lower-limb active orthoses is presented in a recent study [26], to identify locomotion-related activities of daily living in healthy or mildly impaired people with residual movement capabilities. The algorithm is based on a fuzzy-logic classifier operating on signals acquired from the onboard mechanical sensors (hip joint potentiometers) and an IMU (for foot contact detection) integrated in the backpack of a fully portable hip exoskeleton. The LMR is performed with an overall accuracy rate of $97.4 \%$. Despite the high accuracy rate, the classifier was tested only for self-selected cadence and under the zerotorque mode of the orthosis. The kinematics alteration induced by the user's walking pace or the robot assistive actions were misregarded in the current state of the art.

This study aims to design a control architecture for seamlessly detecting locomotion-related activities of daily living in real-time, with the goal of overcoming existing limitations. The controller is implemented on a fully autonomous active pelvis orthosis (APO) and can distinguish between seven main locomotion-related activities of daily living (i.e., ground-level walking, stair ascending, stair descending, sitting, standing, sit-to-stand, and stand-to-sit). Sensory information is acquired from onboard hip joint encoders and plantar pressure sensitive insoles worn by the user and communicating wirelessly with the cognitive unit of the APO. A hybrid approach is chosen. This approach is a simple time-based decoding process responsible of distinguishing between static and dynamic tasks relying only on the hip joint kinematics. An event-based approach is used to classify among dynamic modes (i.e., walking, stair ascending, and descending). The task classification is computed at a certain event of the gait cycle, i.e. the foot strike. Instead of avoiding the use of additional sensors, we exploit a remarkable gait feature that can be acquired using plantar pressure sensors, i.e., center of pressure $(\mathrm{CoP})$. At each foot strike, the features extracted for classification, namely the hip joint angles and the $\mathrm{CoP}$ position, are clearly different among tasks. Three membership regions in the space described by the extracted features are used to classify the tasks in a subject-independent fashion every time the foot strike event is detected. An experimental protocol is designed to assess the feasibility and validate the effectiveness of the proposed LMR approach in an out-of-lab scenario. Furthermore, its robustness is validated in a human in-the-loop application (i.e., when assistive mechanical power is transferred from the robot to the user on top of the LMR process).

The proposed LMR method is reported in Section II along with the mechatronic overview on the APO and the experimental protocol. The experimental results are shown in Section III and discussed in Section IV. The conclusions are drawn in Section V.

\section{LOCOMOTION MODE RECOGNITION ALGORITHM}

The LMR algorithm and its integration in the control architecture of the APO are described in this section. The experimental activities performed to validate the proposed LMR method are then reported.

\section{A. Locomotion mode recognition}

The LMR algorithm is an advancement of the method presented in our previous works [30], [31]. Hereafter, its theoretical formulation and working principle are described. The LMR algorithm can identify seven main locomotion modes of daily life activities, namely, standing still (standing), sitting (sitting), sit-to-stand, stand-to-sit, ground-level walking (GLW), ascending stairs (AS), and descending stairs (DS). The seven locomotion modes are first divided into three classes as follows: static, transient, and dynamical. The static modes include sitting and standing. The transient modes include the transitions between sitting and standing (i.e., sit-to-stand and stand-to-sit). The dynamical modes include GLW, AS, and DS. For the static modes, the transition between sitting and standing is bidirectional and can be achieved through the states sit-to-stand and stand-to-sit respectively. For the dynamical modes, the transition between GLW and AS/DS is bidirectional, while that between AS and DS is prohibited. The transition between the static and dynamical modes can only be achieved by passing from the standing state to each dynamical mode, and vice versa (Fig. 1).

1) Static and transient mode recognition: Note that during the static modes, the two legs move together. During dynamical modes, instead, the two legs move in counterphase. A time-based approach was selected to differentiate the dynamical modes from the static ones, operating on the right and left hip joint trajectories as follows: the relative angle $\theta_{L R}$ between the left hip angle $\theta_{L}$ and the right hip angle $\theta_{R}$ was first computed:

$$
\theta_{L R}=\left|\theta_{L}-\theta_{R}\right|
$$

The $\theta_{L R}$ values during a period of $\Delta_{t 1}$ were then buffered. The static mode was determined if the buffered values were smaller than the threshold value $\theta_{\text {Tstatic }}$; otherwise, the mode was determined to be a dynamical one. In static modes, $\theta_{L R}=0$ deg. $\Delta_{t 1}$ should be long enough to neglect a wrong classification during those phases of dynamical modes in 


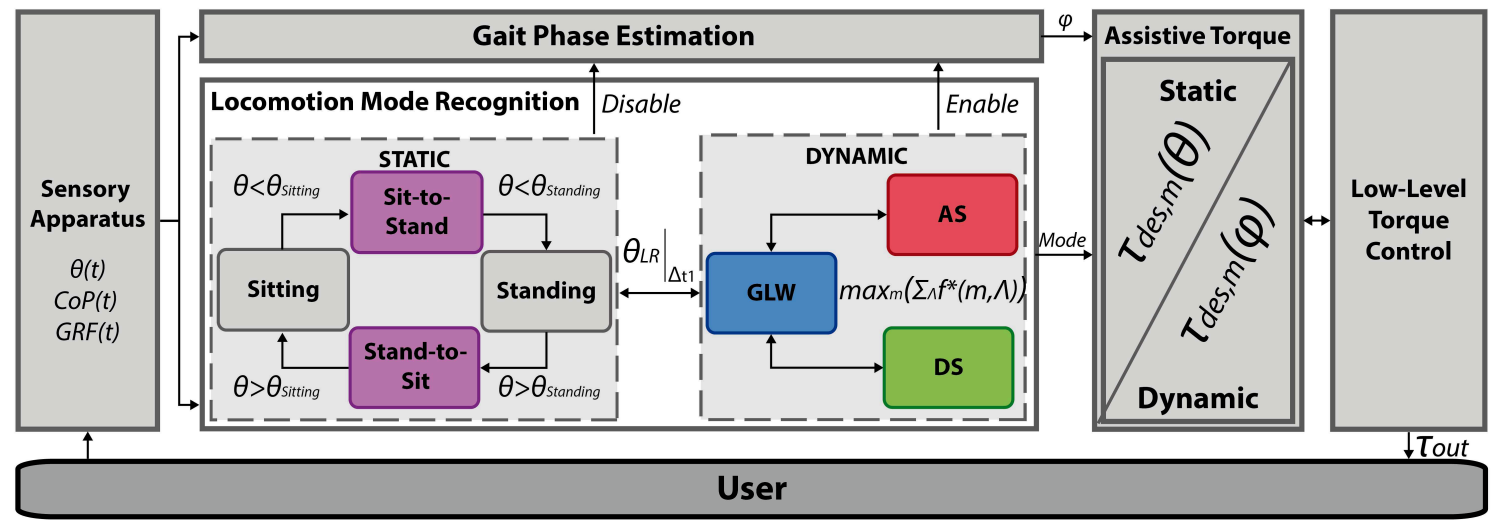

Fig. 1. Schematic block diagram of the control architecture. Kinematic and kinetic variables are collected from the sensory apparatus and given as input to the LMR block. The LMR block first classifies static and dynamic modes, then identifies the specific mode. In parallel, the gait-phase estimation block provides an estimate of the cyclical phase of the dynamical modes. The decoded activity is responsible of generating the assistive torque reference.

which the hip angles were similar (i.e., the transition accuracy would be lowered by small values), still preserving a fast computation, otherwise delayed by higher values. In this work, $\Delta_{t 1}$ and $\theta_{T \text { static }}$ were chosen on a statistical basis as $200 \mathrm{~ms}$ and $10 \mathrm{deg}$, respectively. Within the static modes, the transition within the transients sit-to-stand/stand-to-sit was managed with a simple threshold mechanism. The hip joint angle during sitting was approximately $90 \mathrm{deg}$, whereas that during standing was approximately $0 \mathrm{deg}$. The threshold values $\theta_{\text {Standing }}$ and $\theta_{\text {Sitting }}$ were responsible for the state transitions and set as 80 deg and $20 \mathrm{deg}$, respectively. They were applied on the mean value between the right and left limbs as $\theta_{\text {Sitting }}<\left(\theta_{L}+\theta_{R}\right) / 2<$ $\theta_{S \text { tanding }}$ and represented the onset of a transition. The jumps in the transient states were reversible, which meant that the state machine was allowed to enter and exit from the state without necessarily moving in the succeeding one whenever the person tries to stand up/sit down without completing the motion.

2) Dynamical modes: The transition between different dynamical modes (i.e., GLW, AS, and DS) relied on an eventbased approach. The event was selected as the foot-strike (i.e., when the foot initially strikes the ground) and detected by the sensorized insoles with a threshold-based method imposed on the vertical ground reaction force (vGRF). The foot strike was chosen for two main reasons: 1) it can easily be detected using sensorized shoes and 2) its detection was used to sample three gait features that behaved differently depending on the locomotion task (Fig. 2), thereby being effective in performing a subject-independent classification. Two variables related to locomotion tasks were used to acquire the gait features, namely the hip joint angles measured by the onboard encoders of the APO and the CoP measured using the instrumented sensorized shoes. The first feature was defined as the hip angle of the proceeding limb at the foot strike $\left(\theta_{f s k}\right.$ and named as $\left.\wedge_{1}\right)$. Taking $\theta_{f s k}$ in GLW as a reference value, this angle increased in AS because the hip flexion to lift and place the foot was higher. It decreased during DS because the stair length limited the flexion range of the hip joint. The second feature was defined as the $\mathrm{CoP}$ of the proceeding limb at the footstrike $\left(\mathrm{CoP}_{f s k}\right.$, which is also called $\left.\wedge_{2}\right)$. During GLW, the heel usually strikes the ground before the toe, and the CoP moves forward from the rear foot to the forefoot. Hence, the $C o P_{f s k}$ was at the rear foot. For the locomotion on stairs, in addition to the foot inclination, the CoP was also influenced by the stair length, which limited the foot placement. As a result, the foot usually strikes the ground with the middle and fore parts. The third feature, namely the contralateral hip joint angle at the foot strike of the proceeding limb $\left(\theta_{f s k}^{C L}\right)$, was included in the classification process to improve the reliability of the algorithm and its robustness to inter-subject variability. In GLW, $\theta_{f s k}^{C L}$ indeed assumed negative values, with the hip angle being in the extended configuration. The feature during AS and DS was positive, with the contralateral leg not being completely extended. A non-linear transformation around $0 \mathrm{deg}$ was applied to enhance the difference among the feature in different modes and emphasize the distinction between the positive and negative values (i.e., the arctangent transformation $\left.\arctan \left(\theta_{f s k}^{C L}\right)\right)$. As a result, $\arctan \left(\theta_{f s k}^{C L}\right)$, also named as $\wedge_{3}$, was selected as the third feature. Three regions corresponding to the three dynamical modes were identified in a 3D space described by the extracted features. A fuzzy logic recognition method was proposed to classify the dynamical modes. Each region was described by a linear combination of three membership functions (one for each feature). The membership function computed the membership value for the event-based feature to belong to the target mode. The membership was defined in the range $\left(\begin{array}{ll}0 & 1\end{array}\right], 1$ being the maximum membership to belong to that mode.

The proper set of subject-independent membership functions was identified by performing a preliminary offline analysis on the data collected during dynamical modes in a controlled environment for a secure data collection. 50 strides in GLW, AS, and DS were collected from six subjects (different from the participants to the experimental activity of this work) to estimate the mean value $\mu_{m, \wedge}$ and the standard deviation $\sigma_{m, \wedge}$ for a set of generalized membership functions gener- 

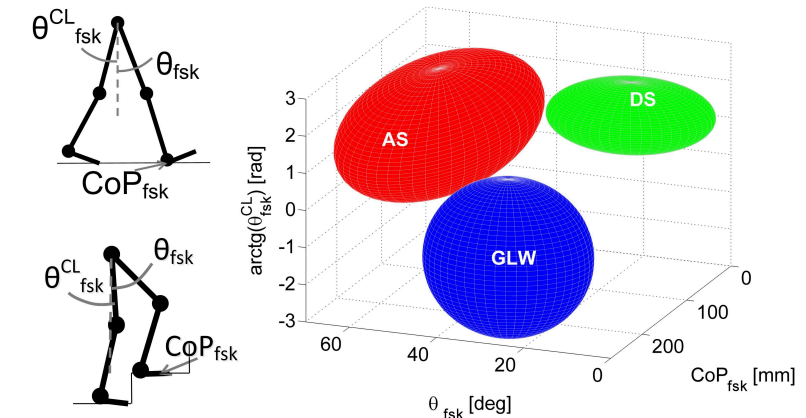

(a)
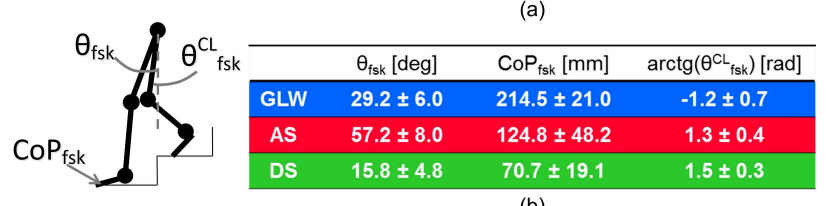

(b)

Fig. 2. (a) 3D plot of the generalized membership functions $f(m, \Lambda)$ of the three features $\Lambda$. Each ellipsoid represents the membership region of a specific dynamic mode $m$ in the space of coordinates $\Lambda$. (b) Schematic table reporting values for $\mu_{m, \Lambda}$ and $\sigma_{m, \Lambda}$. Each column reports a feature $\Lambda$, while each line reports a mode $m$.

ated for the real-time LMR of the dynamical modes. Fig.2 shows an overview of the membership functions generated from the offline data sets. The membership functions of the feature values for each task were approximated as a Gaussian distribution $N\left(\mu, \sigma^{2}\right)$, where $\mu$ and $\sigma$ are the mean value and the standard deviation, respectively. Fig. $2 \mathrm{~b}$ presents the values of $\mu_{m, \wedge}$ and $\sigma_{m, \wedge}$ for different memberships. Given the vector $m=(G L W A S D S)^{T}$, the membership function of GLW for the first feature $\wedge_{1}$ was designed as follows:

$$
f\left(m_{1}, \wedge_{1}\right)=k_{1} N\left(\mu_{m_{1}, \wedge_{1}}, \sigma_{m_{1}, \wedge_{1}}^{2}\right)
$$

where $\mu_{m_{1}, \wedge_{1}}$ and $\sigma_{m_{1}, \wedge_{1}}$ are the mean value and the standard deviation of $\theta_{f s k}$ during GLW, respectively. $k_{1}$ is the scaling factor that normalizes the maximal value of $N\left(\mu_{m_{1}, \wedge_{1}}, \sigma_{m_{1}, \wedge_{1}}^{2}\right)$ to be 1. Scaling the Gaussian distributions allowed to assign the same weight to the membership functions of different features independently on their inherent variability. The feature values during AS/DS also had similar distributions, and as described before, the mean feature value during DS $\mu_{m_{3}, \wedge_{1}}$ was smaller than $\mu_{m_{1}, \wedge_{1}}$. During AS, $\mu_{m_{2}, \wedge_{1}}$ was larger than $\mu_{m_{1}, \wedge_{1}}$. Note that the mode with the feature value $\wedge_{1}$ greater than $\mu_{m_{2}, \wedge_{1}}$ was most likely to be AS. Hence, the membership function of the AS was designed as:

$$
f\left(\mu_{m_{2}, \wedge_{1}}\right)= \begin{cases}k_{2} N\left(\mu_{m_{2}, \wedge_{1}}, \sigma_{m_{2}, \wedge_{1}}^{2}\right), & \theta_{f s k}<\mu_{m_{2}, \wedge_{1}} \\ 1, & \theta_{f s k} \geqslant \mu_{m_{2}, \wedge_{1}}\end{cases}
$$

Similarly, the mode with the feature value $\wedge_{1}$ lower than $\mu_{m_{3}, \wedge_{1}}$ was most likely to be DS. Its membership function was designed as follows:

$$
f\left(\mu_{m_{3}, \wedge_{1}}\right)= \begin{cases}1, & \theta_{f s k}<\mu_{m_{3}, \wedge_{1}} \\ k_{3} N\left(\mu_{m_{3}, \wedge_{1}}, \sigma_{m_{3}, \wedge_{1}}^{2}\right), & \theta_{f s k} \geqslant \mu_{m_{3}, \wedge_{1}}\end{cases}
$$

The membership functions of the second $\mathrm{CoP}_{f s k}$ and third $\arctan \left(\theta_{f s k}^{C L}\right)$ features were designed in a similar manner. Fig. 2a shows that the three membership regions in the 3D space generated three ellipsoids, whose centers were in the coordinates of the mean value $\mu_{m, \Lambda}$ for the features $\Lambda$ of the mode $m$, and their radii in the three dimensions corresponded to $3 \cdot \sigma_{m, \Lambda}$ for the features $\Lambda$ of the mode $m$. The membership grade was a function of the proximity to the center of each ellispoid following a Gaussian distribution dependency. The algorithm calculated the membership grade for all features to belong to the mode $m$. The total membership of a specific mode $F(m)$ was defined as the sum of the three membership grades:

$$
F(m)=\sum_{i=1}^{3} f\left(m, \wedge_{i}\right)
$$

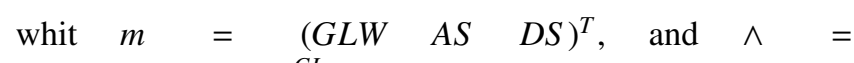
$\left(\theta_{f s k}, \operatorname{CoP} P_{f s k}, \arctan \left(\theta_{f s k}^{C L}\right)\right)$.

Lastly, the target mode $M_{\text {target }}$ was determined as the one with the maximal membership:

$$
M_{\text {target }}=\arg \max _{m_{i}}(F(m))
$$

\section{B. Wearable robotics platform}

The APO, on which the LMR algorithm was implemented, was an advanced prototype of the laboratory tethered platform presented in [27]. The APO, with a CAD model given as a detail of Fig. 4, was a battery-operated orthosis for assisting hip flexion-extension movements. Its mechanical structure comprised a main carbon fiber plate connecting the exoskeleton to an orthopedic cuff enveloping the users trunk. Two carbon fiber lateral extensible arms were endowed with three degrees-offreedom (DoFs) collocated with their anatomical counterpart (i.e., a passive hip adduction-abduction DoF, a passive hip internal-external rotation DoF and an active hip flexionextension DoF). The actuation units (one for each lateral arm) were based on the series elastic actuation (SEA) architecture [28]. Each SEA had a single-axis configuration composed of a motor-reduction stage connected to a torsional spring, whose deformation was directly measured by an absolute encoder. A second encoder placed on the hip axis measured the hip angle.

By means of the DiZic modules integrated in the device, the APO was capable of wirelessly communicating with a pair of shoes instrumented with sensitive insoles via a IEEE 802.15.4 ZigBee communication protocol. The sensitive insoles were composed of a matrix of 64 optoelectronic sensors recording vGRF and the longitudinal position of the CoP [29]. The instrumented shoes were reported as a detail of Fig. 4.

The control architecture of the APO consisted of a hierarchical structure constituted by two layers, namely a lowlevel closed-loop torque control layer and a high-level layer. Low-level closed-loop torque controllers independent for each actuation unit relied on a 2pole-2zero compensator controlling the hip joint output torque. The high-level layer was run at $100 \mathrm{~Hz}$ on a real-time controller sbRIO-9632 (National Instruments, Austin, Texas, US), endowed with a FPGA processor implementing the low-level layer at $1 \mathrm{kHz}$. 

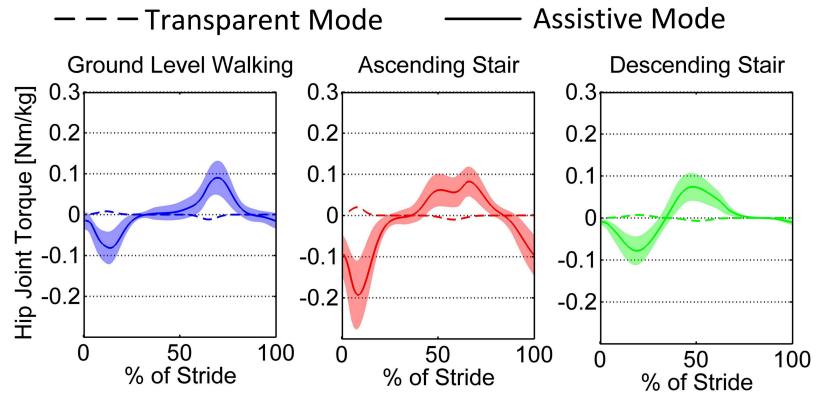

Fig. 3. Assistive profiles delivered by the APO during locomotion-related activities in dynamical modes. Data were collected from correctly identified stride within each mode from all the subjects under the AM utilization. The data were then segmented and re-sampled over the percentage of the gait cycle starting from the foot strike.

\section{Assistive strategy}

An assistive strategy for providing adaptive assistance to the participant while performing locomotion-related activities was developed to validate the proposed classifier under the human-in-the-loop application. The evaluation of the effect of the assistive strategy was beyond the goal of this study. The APO was controlled under zero-torque or transparent mode (TM) when it was not requested to deliver an assistive torque reference (i.e., the desired torque reference was set to $0 \mathrm{Nm}$ ). In the assistive mode (AM), the high-level control layer was designed to provide the user of the APO with a phase-locked assistive torque coherently with the decoded locomotion task. The reader can refer to Fig. 1 for a schematic overview of the adaptive high-level control architecture.

The assistive torque profile for the transient modes was smoothly mapped over the hip joint angles, its maximum amplitude centered in the mean position between the two thresholds, $\theta_{\text {Standing }}$ and $\theta_{\text {Sitting }}$, defining the $0-100 \%$ of the sit-to-stand and stand-to-sit tasks. The amplitude depended on a scaling factor selected for each subject as the $10 \%$ of his/her bodyweight. No torque was provided when the subject was in the sitting or standing state.

During the dynamical modes, the APO delivered a phaselocked torque reference depending on the cyclical phase, which inherently described the dynamical locomotion-related activities. An adaptive gait-phase estimator based on adaptive oscillators presented in [32] was a suitable tool for this purpose. Only dynamical modes were periodic. Hence, their detection enabled the gait-phase estimation. The torque vs. phase profile was obtained by averaging the nominal torques measured at the hip level during the locomotion-related activities reported in the biomechanics scientific literature [33]-[36]. Fig. 3 provides an example of $\tau_{d e s, m}(\varphi)$. The same assistive profile was delivered for each subject to have a repeatable disturbance related to the gait-phase of the ambulatory task, independent from the person. Only the intensity was varied among the participants, with values scaled as $10 \%$ of the bodyweight of each subject.

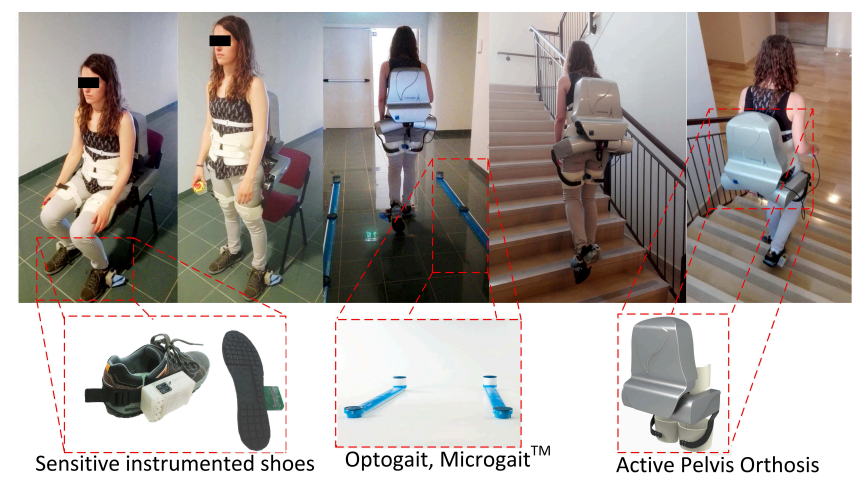

Fig. 4. Experimental setup. The person wearing the APO and the sensitive instrumented shoes was requested to perform sitting, standing, GLW, AS, and DS.

\section{Experimental protocol}

Six healthy subjects (five males and one female) (average height: $1.74 \pm 0.07 \mathrm{~m}$; averaged weight: $73.7 \pm 10.8 \mathrm{~kg}$ ) volunteered to participate in the experimental activities. The experiments were performed at the premises of Fondazione Don Carlo Gnocchi (Florence, Italy). A track was defined, including all the investigated locomotion modes and the possible transitions between them. The controller demonstrated capabilities of managing the transitions between the static and dynamical modes (e.g., those from standing to AS or DS and vice-versa in our previous works) with $100 \%$ success rate [30], [31]. Hence, the track designed for this activity was not inclusive of those transitions. The track consisted of the sequence of the following actions: sitting, sit-to-stand, standing, GLW, AS, GLW, AS, GLW, DS, GLW, DS, GLW, standing, stand-to-sit, and sitting. The sequence of the listed tasks for each track repetition was performed seamlessly transiting between each consecutive mode to include the transitions between different tasks in the investigation. The subjects wore the APO and the sensorized shoes during the whole experiment. Fig. 4 shows an overview of the experimental protocol with a participant wearing the APO and instrumented shoes and performing different locomotion-related activities. Each subject underwent four sessions: (i) they performed the track under TM utilization of the APO at a self-selected pace; (ii) they performed the track under TM utilization at a fast cadence to validate the LMR robustness against an increase of the walking cadence; (iii) for the LMR robustness against a decrease in walking cadence, they performed the track under TM utilization at slow cadence; and (iv) performed the track under AM utilization at a self-selected pace to assess the robustness to the disturbances induced by the mechanical assistive action of the robot. The track was repeated three times during each session. Participants were asked to keep the selected cadences constant during the execution of each track. The cadences were assessed by measuring the walking speed and the step length using an Optogait system (Microgate, Bolzano, Italy) placed in a $10 \mathrm{~m}$ length at the beginning and end of the track in the GLW path. 

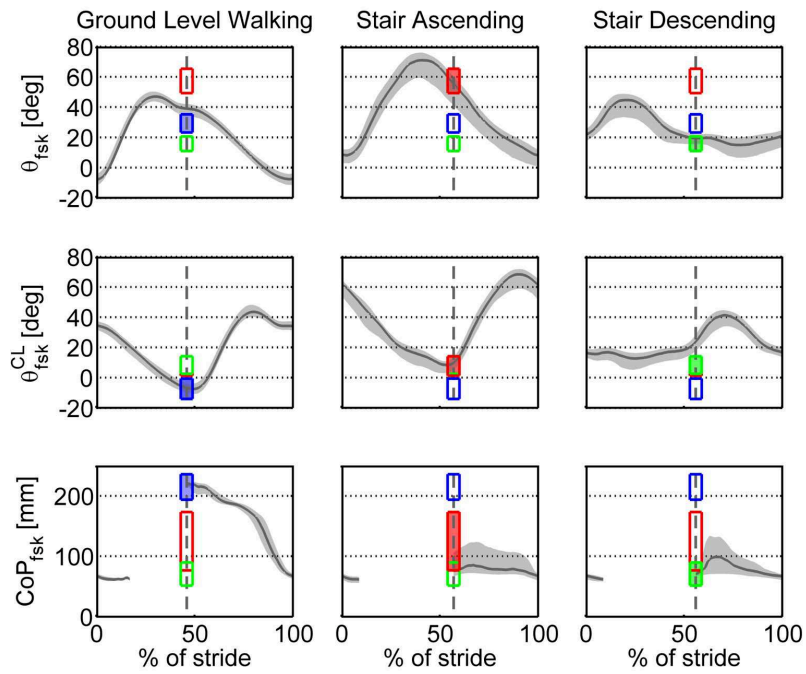

Fig. 5. Kinematic analysis of the variables used for the LMR. Each line reports a feature $\Lambda$, while each column reports a mode $m$. In each graph, the three windows represent the membership function $\left.f_{(} m, \Lambda\right)$. The collected variables (i.e., the hip joint angle and the $\mathrm{CoP}$ of the right and left limbs from all the subjects walking under all the conditions) are segmented at the foot strike and re-sampled over the interval $0-100 \%$ of the stride cycle contoured by the interval between the 25 th and 75 th percentiles. $f(m, \Lambda)$ windows are reported as rectangles with a height equal to $\mu_{m, \Lambda} \pm \sigma_{m, \Lambda}$ and a width equal to the standard deviation of the instant of occurrence of the foot strike. For each column, only the corresponding $\left.f_{(} m, \Lambda\right)$ is filled (blue for GLW, red for AS, and green for DS).

\section{E. Data analysis}

The collected speeds, cadences, and step lengths were analyzed for all the participants to prove differences between the trials performed at different cadences. The collected variables from the APO (i.e., the right and left hip joint angles and the right and the left $\mathrm{CoP}$ ) were segmented for each stride and resampled over the percentage of the gait cycle starting from the foot strike. The LMR performance was evaluated in terms of the accuracy rate (i.e., the number of successful recognition over the total number of strides). The analysis was done at the level of each participant, in each trial of the experimental protocol, and at global level considering the total number of strides from all the participants and all conditions. Finally, the delay in the recognition of a different task was computed (i.e., amount of time elapsed between the initiation and the detection of a new activity).

\section{EXPERIMENTS AND RESULTS}

This section presents the results obtained from the experimental activities.

\section{A. Spatial-temporal and kinematic gait variables}

The spatial-temporal gait variables were averaged for each participant in the three repetitions of each different walking condition. Consistently, each participant varied the walking cadence reducing/increasing the step length together with an increment/decrement of the cadence. The averaged values of the spatial-temporal gait variables resulted in a step length decrease of approximately $18.0 \%$ from $70.89 \pm 4.77 \mathrm{~cm}$ to
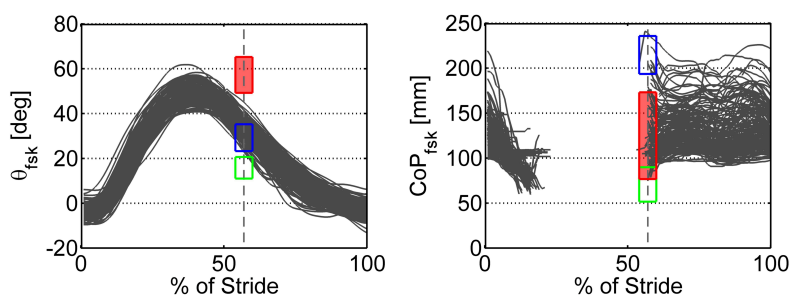

Fig. 6. Detail of the kinematic analysis for Subject\#4 performing AS. The hip joint angle and the $\mathrm{CoP}$ are reported with the windows of the membership function $\left.f_{(} m, \Lambda_{1}\right)$ (on the left) and $\left.f_{(} m, \Lambda_{2}\right)$ (on the right). The collected variables from the left and right limbs in the AS task are segmented at the foot strike and re-sampled as a percentage of the stride cycle. $\left.f_{(} m_{2}, \Lambda\right)$ windows are reported as rectangles with height $\mu_{m, \Lambda} \pm \sigma_{m, \Lambda}$ and width equal to the standard deviation of the instant of occurrence of the foot strike. Only the $\left.f_{(} m_{2}, \Lambda\right)$ is filled (red rectangle). The GLW (blue) and DS (green) are empty.

$58.13 \pm 7.78 \mathrm{~cm}$ when the participants were asked to increase their cadence and increased of $24.6 \%$ to $88.41 \pm 6.98 \mathrm{~cm}$ when they were asked to reduce it. Longer steps resulted in a cadence reduction of $10.4 \%$ from $96.74 \pm 3.58$ steps/min to $86.64 \pm 0.13$ steps/min, whereas shorter steps resulted in an increase of $10.9 \%$ of the cadence to $107.17 \pm 8.18$ steps/min. The same percentage, but with an inverted trend, was recognizable for the walking speed $(12.3 \%$ increase for longer steps and $8.8 \%$ decrement for shorter steps). The spatial-temporal gait variables when walking under the AM were not altered with respect to the zero-torque mode at the self-selected pace.

The kinematic variables were segmented offline and resampled over the stride percentage starting from the foot strike. Fig. 5 shows the kinematic profiles of the hip joint angles averaged for all subjects and conditions. On the same graphs, the interval underlying the generalized membership functions $f_{m, \wedge}$ for each feature $\wedge$ were rectangles centered in their mean value $\mu_{m, \wedge}$ on the y-axis with a height of $\mu_{m, \wedge} \pm \sigma_{m, \wedge}$ and with the mean value of the percentage of the stride cycle of the foot strike occurrence on the X-axis. Fig. 5 illustrates that the expected kinematic variables were not always consistent with the membership functions, which preannounced the occurrence of the wrong detection of the current mode. This is the case for the three following conditions: (i) $\theta_{f s k}$ during GLW in an interval of $39.08 \pm 4.45 \mathrm{deg}(33.9 \%$ higher compared to $\left.\mu_{m_{1}, \wedge_{1}}\right)$; (ii) $\arctan \left(\theta_{f s k}^{C L}\right)$ during DS in the interval $0.45 \pm 0.06$ $\operatorname{rad}\left(69.0 \%\right.$ lower than $\left.\mu_{m_{3}, \wedge_{3}}\right)$; and (iii) $C o P_{f s k}$ of the AS in the interval $75.87 \pm 30.63 \mathrm{~mm}\left(39.5 \%\right.$ lower than $\left.\mu_{m_{2}, \wedge_{2}}\right)$.

\section{B. Recognition accuracy rate}

The recognition accuracy rate within the dynamic modes was defined as the number of accurately recognized steps over the total number of steps accomplished during the dynamical modes. Table I shows an overview of the experiments results.

1) Transparent mode at the self-selected speed: Each subject walked on average for $262 \pm 43$ steps for each track. While the number of steps in GLW changed for each subject with the cadence selected on their preference, the number of steps on the stairs was fixed and equal to 42 , with the number of steps always the same for all the subjects in one trial. The number of seamless transitions between two different consecutive modes 
was eight for each track repetition. The accuracy rate under the TM walking condition at the self-selected speed was $100 \%$ in all the conditions, except for the two cases (i.e., Subject\#1 with one mistaken step in GLW and Subject\#3 with one mistake in DS). As a result, when considering the TM walking at the self-selected speed for all the subjects, only two mistakes were committed with an accuracy rate of $99.9 \%$ for GLW, $100 \%$ on AS, and $99.8 \%$ on DS. Under this situation, the intersubject variability had a negligible effect, if any, on the LMR performance.

2) Transparent mode at the fast and slow cadences: The accuracy rate was slightly affected by the walking cadence. In the case of the slow cadence, the accuracy rate decreased for Subject\#2 (five mistakes in GLW), Subject\#4 (five mistakes in AS), and Subject\#6 (two mistakes in GLW, 1 in AS and 1 in DS). The difference between the mistaken steps for the other subjects was not greater than 1. In general, the accuracy rate in GLW at a slow cadence was $99.4 \%$ over 1317 steps (eight steps against 1 at the self-selected speed). In AS and DS, six mistakes (98.8\% accuracy rate) and one mistake (99.8\%) were counted. In the fast walking cadence, the number of wrong interpretations for the GLW was nine with an accuracy rate of $99.5 \%$. The accuracy rate of the AS was also negatively affected with a number of mistaken interpretations equal to 10 (98.0\%). Only two mistakes were committed for DS (99.4\%). As a major consequence of a different kinematic, only the results for AS were lowered with a corresponding reduction of $1.9 \%$ accuracy rate.

3) Assistive mode at the self-selected speed: Under this condition, the results were not altered for GLW and DS (two mistakes in both modes) with a similar accuracy rate as for TM at the self-selected speed. Errors did not occur for a kinematic alteration of the hip joint angles induced by the mechanical power transfer. The mode that was more significantly mistaken was still the AS (13 mistakes for a corresponding accuracy rate of $97.4 \%$ ). For most of the subjects, the results were not significantly affected (one mistake in DS for subjects \#1 and \#3, one in GLW for Subject\#5, and one in AS for Subject\#6). Subject\#4 showed a critical decrease of the accuracy rate with a total of one mistake in GLW, one in DS, and 12 for AS. Consequently, under AM, the global accuracy rate was lowered by $2.6 \%$.

4) Global results: The accuracy rate for GLW on the total count of steps was $99.7 \%$ for 6412 steps, resulting in the misclassification of only 21 steps. The accuracy rate for the DS mode was $99.6 \%$ over 2016 steps (i.e., only eight in total). The worst case was the AS mode with an accuracy rate of $98.6 \%$ over 2016 steps (i.e., 29 mistaken steps). The main reason for a poor LMR accuracy rate was related to a huge source of mistaken tasks in Subject\#4. The subject was the tallest $(1.88 \mathrm{~m})$, and the accuracy rate of AS was very poor. The total number of mistaken steps in AS for Subject\#4 amounted to 27 steps (over a total of 29). The accuracy rate for AS if Subject\#4 was not included in the analysis would have been $99.8 \%$. The same happened in GLW. Subject\#4 has a total of 10 mistaken steps (over 21). Excluding Subject\#4 from the analysis, the accuracy rate for GLW was $99.8 \%$. Fig. 6 shows that the main sources of wrong detection were provided by a lower $\theta_{f s k}$ in AS compared to the expected $f_{m_{2}, \wedge_{1}}$ and attributable to a high $\operatorname{CoP}_{f_{s k}}$ that can be confused with the $f_{m_{2}, \wedge_{2}}$. More importantly, the total number of performed transitions between two consecutive dynamical modes during the experimental activities was 96 for each subject (576 in total) (i.e., almost $5.5 \%$ of the total number of performed steps). None of the misclassification occurred in the correspondence of a transition. Finally, considering the total number of steps performed independently on the mode (10444) and the total number of mistaken steps (58), the global accuracy rate of the proposed LMR architecture was $99.4 \%$. The transitions between the static and dynamic modes and

TABLE I

RESULTS OF THE LMR PROCESS.

\begin{tabular}{|c|c|c|c|c|c|c|c|c|c|c|c|}
\hline & & \multicolumn{2}{|c|}{ TM } & \multicolumn{2}{|c|}{ TM Slow } & \multicolumn{2}{|c|}{ TM Fast } & \multicolumn{2}{|c|}{$\mathrm{AM}$} & \multicolumn{2}{|c|}{ All Modes } \\
\hline & & $\begin{array}{l}\text { Number } \\
\text { of steps }\end{array}$ & $\begin{array}{l}\text { Accuracy } \\
\text { rate }(\%)\end{array}$ & $\begin{array}{l}\text { Number } \\
\text { of steps }\end{array}$ & $\begin{array}{l}\text { Accuracy } \\
\text { rate }(\%)\end{array}$ & $\begin{array}{l}\text { Number } \\
\text { of steps }\end{array}$ & $\begin{array}{l}\text { Accuracy } \\
\text { rate }(\%)\end{array}$ & $\begin{array}{l}\text { Number } \\
\text { of steps }\end{array}$ & $\begin{array}{l}\text { Accuracy } \\
\text { rate }(\%)\end{array}$ & $\begin{array}{l}\text { Number } \\
\text { of steps }\end{array}$ & $\begin{array}{c}\text { Accuracy } \\
\text { rate }(\%)\end{array}$ \\
\hline \multirow{3}{*}{ Subject\#1 } & GLW & 279 & 99.64 & 236 & 100.00 & 368 & 100.00 & 293 & 100.00 & 1176 & 99.91 \\
\hline & AS & 84 & 100.00 & 84 & 100.00 & 84 & 100.00 & 84 & 100.00 & 336 & 100.00 \\
\hline & DS & 84 & 100.00 & 84 & 100.00 & 84 & 100.00 & 84 & 98.81 & 336 & 99.70 \\
\hline \multirow{3}{*}{ Subject\#2 } & GLW & 284 & 100.00 & 222 & 97.75 & 328 & 100.00 & 286 & 100.00 & 1120 & 99.55 \\
\hline & AS & 84 & 100.00 & 84 & 100.00 & 84 & 100.00 & 84 & 100.00 & 336 & 100.00 \\
\hline & DS & 84 & 100.00 & 84 & 100.00 & 84 & 100.00 & 84 & 100.00 & 336 & 100.00 \\
\hline \multirow{3}{*}{ Subject\#3 } & GLW & 264 & 100.00 & 222 & 100.00 & 358 & 99.72 & 278 & 100.00 & 1122 & 99.91 \\
\hline & AS & 84 & 100.00 & 84 & 100.00 & 84 & 100.00 & 84 & 100.00 & 336 & 100.00 \\
\hline & DS & 84 & 98.81 & 84 & 100.00 & 84 & 100.00 & 84 & 98.81 & 336 & 99.40 \\
\hline \multirow{3}{*}{ Subject\#4 } & GLW & 229 & 100.00 & 198 & 100.00 & 305 & 97.38 & 250 & 99.20 & 982 & 98.98 \\
\hline & AS & 84 & 100.00 & 84 & 94.05 & 84 & 88.10 & 84 & 85.71 & 336 & 91.96 \\
\hline & DS & 84 & 100.00 & 84 & 100.00 & 84 & 100.00 & 84 & 98.81 & 336 & 99.70 \\
\hline \multirow{3}{*}{ Subject\#5 } & GLW & 271 & 100.00 & 226 & 99.56 & 261 & 100.00 & 277 & 99.64 & 1035 & 99.81 \\
\hline & AS & 84 & 100.00 & 84 & 100.00 & 84 & 100.00 & 84 & 100.00 & 336 & 100.00 \\
\hline & DS & 84 & 100.00 & 84 & 100.00 & 84 & 100.00 & 84 & 100.00 & 336 & 100.00 \\
\hline \multirow{3}{*}{ Subject\#6 } & GLW & 243 & 100.00 & 213 & 99.06 & 270 & 100.00 & 251 & 100.00 & 977 & 99.80 \\
\hline & AS & 84 & 100.00 & 84 & 98.81 & 84 & 100.00 & 84 & 98.81 & 336 & 99.40 \\
\hline & DS & 84 & 100.00 & 84 & 98.81 & 84 & 96.43 & 84 & 100.00 & 336 & 98.81 \\
\hline \multirow{3}{*}{ All } & GLW & 1570 & 99.94 & 1317 & 99.39 & 1890 & 99.52 & 1635 & 99.82 & 6412 & 99.67 \\
\hline & AS & 504 & 100.00 & 504 & 98.81 & 504 & 98.02 & 504 & 97.42 & 2016 & 98.56 \\
\hline & DS & 504 & 99.80 & 504 & 99.80 & 504 & 99.40 & 504 & 99.40 & 2016 & 99.60 \\
\hline
\end{tabular}


that between the sit-to-stand and stand-to-sit tasks (i.e., the transient in between the standing and sitting conditions) were always detected correctly for all the subjects, thereby resulting in a $100 \%$ accuracy rate. The critical parameter in this sense was the delay of the recognition reported in the following subsection.

\section{Recognition delay during transitions}

The delay of the recognition process when transiting from two different consecutive tasks is presented in this section. In the case of a transition within the dynamical modes, the delay was defined as the time elapsed from the beginning of the execution of a certain task and the time of its detection. Taking the instant of the initiation as the one in which the limb approaching the new terrain starts the swing phase for reaching the new mode, the results can be easily interpreted as number of steps before the recognition, with the LMR output updated at each foot strike. As a result, the delay between two modes was always one step. The delay between two consecutive modes in the static condition was defined as the ratio between the time elapsed at the detection of the transition over the total time window of accomplishment of that transition. The variables collected from each subject were averaged and resampled over the percentage of accomplishment of the sitto-stand/stand-to-sit task. Fig. 7(a) shows a graph reporting the transient execution. The average duration of the sit-tostand and stand-to-sit actions was $0.89 \pm 0.12 \mathrm{~s}$ and $1.02 \pm 0.16$ $\mathrm{s}$, respectively. The delay from the initiation of the sit-tostand/stand-to-sit task action and the detection of the task execution was evaluated. The analysis was performed on the whole data set without distinguishing between specific conditions. The results showed a delay of $36.2 \%$ in recognizing the sit-to-stand task on its total execution time and $15.2 \%$ for the stand-to-sit. The percentage of time effectively spent in the transient window over the total time spent passing from sitting to standing and vice-versa was also computed. The transient of the sit-to-stand action occupied a window of $31.70 \%$ of the task execution, while that of stand-to-sit action was as long as $50.40 \%$. The time window during which the controller of the APO was providing assistive action for aiding the transients between the sitting and standing actions can be derived from these data. Fig. 7(b) shows an example of the delivered torque during transients. Finally, the analysis was performed for the transitions between the static and dynamic modes. The average delay that existed between the gait initiation and the detection of the dynamical mode and the gait termination and the detection of the static mode was computed. For the first analysis, the delay was computed as the instant in which the leading limb started moving and the instant in which the limb was back in the same position (i.e., the time of the first step). The result of this analysis showed a delay of $60.6 \%$ in detecting the dynamic mode from the gait initiation over the duration of the first step. The delay at the gait termination was computed as the time between the last step of the contralateral limb, ending the gait, and the instant of the detection of the static mode. In line with the previous result, the delay in this case was of $63.8 \%$.

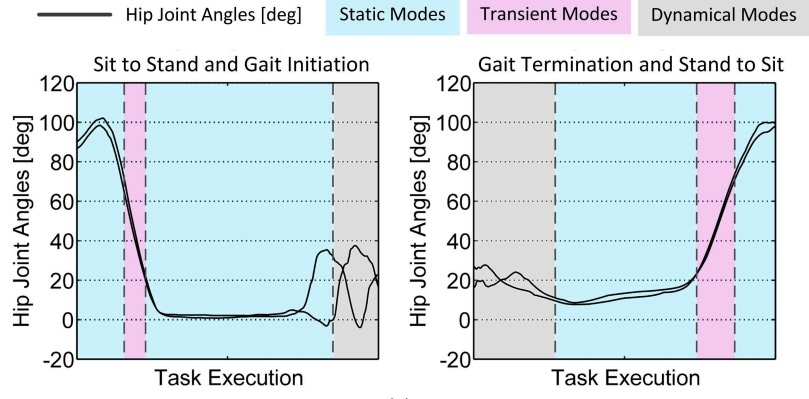

(a)
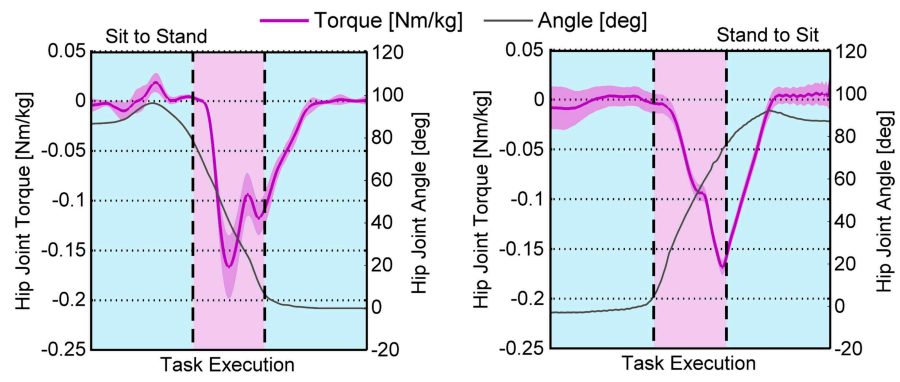

(b)

Fig. 7. (a) Transition within the transients in the static modes and from the static modes to the dynamical modes. The black lines are the right and left hip joint angles from Subject \#3. The blue windows represent the static modes, while the purple windows represent the transients. The gray windows represent the dynamical modes. The gray dotted lines are placed in the instant of the transition detection. (b) Assistive profile delivered during sit-to-stand and stand-to-sit actions. The data are re-sampled over the percentage of the task completion indicated by the two vertical dotted black lines. The gray line shows the average angle profiles during the transient, while the purple line denotes the delivered torque.

\section{Discussion}

This contribution presented a novel approach for the LMR of locomotion-related activities of daily living to provide assistance to the user of an APO. The subject independency of the kinematic and kinetic features extracted at the foot strike event underlies the novelty of the proposed method. The control architecture was validated in an experimental protocol aimed at evaluating the performance of the LMR method and proving robustness to: (i) anthropometries and inter-subject variability, (ii) different walking cadences, and (iii) human-inthe-loop application.

\section{A. Spatial-temporal gait variables}

During the experimental activities, the kinematic data collected from the orthosis and from the plantar pressure sensitive insoles were consistent with the gait biomechanics reported in well-established literature of the gait biomechanics [36]. Despite the numerous walking conditions and wide subject anthropometries, the gait features were not spread with high variability, except from the $\operatorname{CoP}_{f s k}$ during AS. In line with previous kinematic studies, this result was a consequence of the higher variability of the foot inclination at the foot contact during AS [33], [34]. The spatial-temporal gait variables showed consistent trends between the subjects when walking under different conditions (i.e., higher or lower cadence compared to the self-selected one) [36]. 


\section{B. Performance of the LMR process}

The experimental activities demonstrated the feasibility of a subject-independent real-time controller for the classification of seven locomotion-related activities relying on the mechanical wearable sensors. The classification was successful in all the explored conditions in the out-of-lab scenario with a global accuracy rate of $99.4 \%$. This result was confirmed by the fact that the classification capabilities were not affected by the presence of the narrow curvatures of the performed track. As expected, despite minor differences in the hip angle kinematics, the foot strike detection is invariant of the gait cycle, therefore negotiation of different cadences did not affect the recognition performance. While the DS and GLW modes showed a minimal decrease, the major consequence of a different walking pace was given for AS. Nevertheless, this behavior had to be linked to Subject\#4 and to the high variability of his $\operatorname{CoP}_{f s k}$. As a second main outcome of this work, the feasibility of the human-in-the-loop application was proven. The algorithm performance was not affected by the mechanical disturbance introduced by the physical interface of the robot, proving the usability of the LMR method in assisted scenarios. The accuracy rate under AM lowered by 2.6\%. More importantly, the errors under this condition did not occur because of the biomechanical alteration of the collected variables induced by the mechanical power transfer. The presented approach showed a performance that was superior to the effective methods proposed in the literature for active prosthetic devices [6]-[8], in which the accuracy rate reached a maximum of $97.7 \%$ in [10]. The accuracy rate proximal to $99 \%$ was reached by fusing mechanical sensing with sEMG interfaces [11], [12]. Nevertheless, the sEMG interfaces were prone to fade in a prolonged utilization because of the variations of the skin condition, thereby lowering the accuracy rate in long-term use. With respect to the methods based only on mechanical sensing, our results were higher than those from approaches employing threshold-based finitestate machines [14]. The kinematics inter-subject variability can indeed affect the LMR performance, with thresholding being inherently sensitive to minor variations of the recorded kinematics. A work similar to ours was presented in the literature for an active pelvis orthosis [26]. Our controller demonstrated a higher performance. The averaged accuracy rate in [26] was $97.4 \%$ (i.e., $2 \%$ lower than our global accuracy rate of $99.4 \%$ ). Moreover, it is worth noting that in [26], the algorithm was not tested in a human-in-the-loop application. This achievement underlies the use of wearable plantar pressure insoles, as explained in the next subsections.

\section{Wrong detections and recognition delay}

In the perspective of assisted locomotion-related activities, the controller was asked to deliver a different torque profile at the hip level whenever an error occurred in the recognition of a dynamic task. Despite the discrepancy in the torque delivery, the assistive torque profile's shape was characterized by two torque bursts: extension torque and flexion torque. Reasonably, the cyclical behavior of the hip joint over the gait cycle was invariant for the three different modes. Thus, the sequence of extension and flexion torques landmarked with the foot strike was consistent in all the three dynamic modes and changed only in intensity and the timing expressed as a percentage of the gait cycle. Despite being uncomfortable, the perturbation did not represent a real risk of instability. In line with what was stated in [37], the wrong detections of the LMR can be considered critical only when the alteration of mechanical work generated at the level of the actuated joint differed over a certain tolerance range from the expected one during the steady execution of a given mode. Thus, if the variation is within an acceptable range, the error can be considered uncritical for the overall acceptability. Accordingly, none of the participants reported discomfort in the case of an incorrect torque delivery. Envisaging a future application of this integrated approach, the occurrence of misclassification can undermine the effectiveness of assisted locomotion activities both in terms of biomechanics and metabolic cost of the wearer. Moreover, only healthy subjects participated in this work; improper flexion/extension torque delivery in people affected by gait disorders could result in more insidious consequences such as instability and loss of balance.

As in other studies implementing an event-based terrain recognition [15], [16], the maximum delay in a transition between two different modes was $100 \%$ (i.e., occurrence of the next foot strike for the recognition of two consecutive dynamic modes). In contrast to the sEMG-based interfaces [10]-[12], our approach did not possess predictive capabilities in the transitions between modes. In [38], the critical time of the detection of a mode transition was identified as the instant, in which the foot of the contralateral limb approaching the new terrain left the ground at the toe-off moment. In our work, the delay in the transition detection in the dynamical mode was fixed and equal to one step. In other words, the transition will be detected when the proceeding limb has already approached the new terrain, but it will be valid for the subsequent step. While such a lag in the recognition of the new mode can be considered acceptable for healthy users of the powered APO, this framework would not be valid for recognition using a powered lower-limb prosthesis. Indeed, a powered prosthesis should own predicting skills in order to promptly detect the occurrence of a different terrain before the foot strike [38]. Implementation of this method on lower-limb prosthesis would require, beyond identification of input signals different from the hip joint angles measured by the robot encoders, the anticipation of the recognition instant during the swing phase of the proceeding limb.

\section{Limitations of the study}

Despite the promising results, some limitations still have to be pointed out. The main claim of the algorithm is the robustness to inter-subject variability. The results from Subject\#4, who is the tallest participant in our experiment, showed higher failures in identifying his movement intentions possibly because of the widespread variability of his $C_{o} P_{f s k}$ in AS. Even when adaptive strategies were adopted to match the membership function for the hip kinematics, this action should be intended as subject-specific, such that it would reduce the 
claim for subject independency.

The second main limitation is the need for a distributed wearable sensory apparatus, which limits the self-containment of the system. In this framework, the method of [26] remains superior to ours. An integrated gyroscope allows to perform an on-line LMR without the need of any other external sensory apparatus, with the sensory information being acquired from the device itself. The need for plantar pressure sensors is not optimal for the user's acceptability, but still fundamental for preserving the achieved success rates. As an example, the removal of the $\operatorname{CoP}_{f s k}$ would result in a $32.9 \%$ success rate of the AS task for Subject\#4. The overall accuracy would considerably decrease: under all tested conditions, $65.7 \%$ over 6412 steps, $32.9 \%$ over 2016 steps, and $91.2 \%$ over 2016 would be correctly classified for GLW, AS, and DS, respectively. Ensuring high success rate of the LMR method would be fundamental for people with gait impairments for whom a misclassification and delivery of torque related to a different task, could lead to severe consequences such as loss of stability or even falling. This criticality confirms the need for the pressure sensitive insoles.

Despite the wide spectrum of investigated locomotionrelated activities, this study suffered from the absence of two important locomotion modes from the classifier design i.e., up-hill and down-hill walking. The development of suitable strategies for implementing the classification of slope walking will be the object of future studies.

The validation was performed enrolling only healthy participants with a regular gait pattern. The algorithm could be prone to failure in the presence of an altered biomechanics because of pathological gait syndromes. Notably, the definition of subjectspecific, or more generally, impairment-specific membership functions in the case of the pathologic gait will be the most effective strategy to assist subjects according to individual needs and specific gait parameters.

\section{Conclusions}

A major open issue in the field of wearable robotics is the design of control architectures capable of decoding the user's intention in real-time and translating them in viable assistive actions at the level of the actuated joints. This work presented a novel method for the LMR of locomotionrelated activities of daily living and conducted a validation using a fully autonomous APO in out-of-lab settings. The LMR process combined kinematic information from the hip joints with a robust subject-independent event-based fuzzy logic approach driven by sensitive instrumented shoes. The algorithm was capable of decoding locomotion-mode related activities with high accuracy rate and acceptable delay without the need for a subject-specific training. High performances were also preserved in human-in-the-loop applications.

A few issues came forth after the experimental session. The device, albeit very reliable in identifying the users' intentions, presented a slightly wrong recognition when dealing with taller subjects. In this perspective, the integration of additional sensors such as an IMU in the backpack of the device can improve the reliability of the recognition method. Furthermore, pathological gait was not considered for validation. Hence, future works will be steered toward the improvement of the robustness and test the architecture with people affected by gait disorders to verify whether the performance for cooperative robot-assisted locomotion-related activities is preserved.

\section{REFERENCES}

[1] World Health Organization (2011). Global health and aging. http :

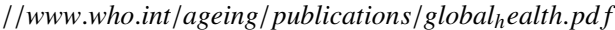

[2] A.M. Dollar and H. Herr, "Lower extremity exoskeletons and active orthoses: challenges and state-of-the-art," IEEE Transactions on robotics, vol. 24, no. 1, pp. 144-158, 2008.

[3] J. L. Pons, "Wearable robots: biomechatronic exoskeletons," John Wiley \& Sons, 2008.

[4] M. R. Tucker et al., "Control strategies for active lower extremity prosthetics and orthotics: A review," J. Neuroeng. Rehabil., vol. 12, no. $1,2015$.

[5] D. Novak and R. Riener, "A survey of sensor fusion methods in wearable robotics," Robot. Auton. Syst., vol. 73, pp. 155-170, 2015.

[6] S. Au et al., "Powered ankle-foot prosthesis to assist level-ground and stair-descent gaits," Neural Networks, vol. 21, no. 4, pp. 654-666, 2008.

[7] H. Huang et al., "A strategy for identifying locomotion modes using surface electromyography," IEEE Trans. Biomedical Engineering, vol. 56, no. 1, pp. 65-73, 2009.

[8] B. Chen et al., "Adaptive slope walking with a robotic transtibial prosthesis based on volitional EMG control," IEEE/ASME Trans. Mechatronics, vol. 20, no. 5, pp. 2146-2157, 2015.

[9] F. Sup et al., "Upslope walking with a powered knee and ankle prosthesis: initial results with an amputee subject," IEEE Trans. Neur. Sys. Reh., vol. 19, no. 1, pp. 71-78, 2011.

[10] D. C. Tkach and L. J. Hargrove, "Neuromechanical sensor fusion yields highest accuracies in predicting ambulation mode transitions for transtibial amputees," Proc of the 35th Annual Int. Conf. of the IEEE Engineering in Medicine and Biology Society, 2013, pp. 3074-3077.

[11] A.J. Young et al., "An intent recognition strategy for transfemoral amputee ambulation across different locomotion modes," Proc of the 35th Annual Int. Conf. of the IEEE Engineering in Medicine and Biology Society, 2013, pp. 1587-1590.

[12] L.J. Hargrove et al., "Robotic leg control with EMG decoding in an amputee with nerve transfers.", New England Journal of Medicine 369.13 (2013): 1237-1242.

[13] M. Gorsic et al., "Online phase detection using wearable sensors for walking with a robotic prosthesis," Sensors, vol. 14, no. 2, pp. 27762794, 2014

[14] L. Ambrozic et al., "CYBERLEGs: A user-oriented robotic transfemoral prosthesis with whole-body awareness control," IEEE Robotics and Automation Magazine, vol. 21, no. 4, pp. 82-93, 2014.

[15] K. Yuan et al., "Fuzzy-logic-based terrain identification with multisensor fusion for transtibial amputees," IEEE/ASME Trans. Mechatronics, vol. 20, no. 2, pp. 618-630, 2015.

[16] B. Chen et al., "A foot-wearable interface for locomotion mode recognition based on discrete contact force distribution," Mechatronics, vol. 32, pp. 12-21, 2015.

[17] T. Yan et al., "Review of assistive strategies in powered lower-limb orthoses and exoskeletons," Robot. Auton. Syst., vol. 64, pp. 120-136, 2014.

[18] P. Beyl et al., "Pleated pneumatic artificial muscle-based actuator system as a torque source for compliant lower limb exoskeletons," IEEE/ASME Trans. Mechatronics, vol. 19. no. 3, pp. 1046-1056, 2014.

[19] M. Cestari et al., "An adjustable compliant joint for lower-limb exoskeletons," IEEE/ASME Trans. Mechatronics, vol. 20, no. 2, pp. 889-898, 2015.

[20] B. Ugurlu et al., "Variable ankle stiffness improves balance control: Experiments on a bipedal exoskeleton," IEEE/ASME Trans. Mechatronics, vol. 21, no. 1, pp. 79-87, 2016.

[21] S. Wang et al., "Design and Control of the MINDWALKER Exoskeleton," IEEE Trans. Neur. Sys. Reh., vol. 23, no. 2, pp. 277-286, 2015.

[22] A. Kilicarslan et al., "High accuracy decoding of user intentions using EEG to control a lower-body exoskeleton," Proc. of the 35th Annual International Conference of the IEEE Engineering in Medicine and Biology Society, 2013, pp. 5606-5609.

[23] J.C. Bradford et al., "Electrocortical activity distinguishes between uphill and level walking in humans," J. Neuropsychol., vol. 115, no. 2, pp. 958966, 2016. 
[24] ReWalk, 2016. http://rewalk.com/rewalk-personal-3/

[25] Ekso Bionics. Ekso Bionics Ekso Product Information. Online. 2014.

[26] J. Jang et al., "Online gait task recognition algorithm for hip exoskeleton," Proc. of the IEEE/RSJ Int. Conf. Intelligent Robots and Systems, 2015, pp. 5327-5332.

[27] F. Giovacchini et al., "A light-weight active orthosis for hip movement assistance." Robot. Auton. Syst., vol. 73, pp. 123-134, 2015.

[28] G. A. Pratt and M. M. Williamson, "Series elastic actuators," Proc. of the IEEE/RSJ Int. Conf. Intelligent Robots and Systems, 1995, pp. 399-406.

[29] S. Crea et al., "A wireless flexible sensorized insole for gait analysis," Sensors, vol. 14, no. 1, pp. 1073-1093, 2014.

[30] K. Yuan et al., "A realtime locomotion mode recognition method for an active pelvis orthosis," Proc. of the IEEE/RSJ Int. Conf. Intelligent Robots and Systems, 2015, pp. 6196-6201.

[31] K. Yuan et al., "Fuzzy-logic-based hybrid locomotion mode classification for an active pelvis orthosis: Preliminary results." Proc. of the 37th Annual Int. Conf. of the IEEE Engineering in Medicine and Biology Society, 2015, pp. 3893-3896.

[32] T. Yan et al., "An oscillator-based smooth real-time estimate of gait phase for wearable robotics," Autonomous Robots, pp. 1-16, 2016.

[33] A. Protopapadaki et al., Hip, knee, ankle kinematics and kinetics during stair ascent and descent in healthy young individuals, Clinical Biomechanics, vol. 22, pp. 203210, 2007.

[34] R. Riener et al., Stair ascent and descent at different inclinations, Gait and Posture, vol.15, pp.3244, 2002.

[35] K. Endo and H. Herr, Human Walking Model Predicts Joint Mechanics, Electromyography and Mechanical Economy, The 2009 IEEE/RSJ International Conference on Intelligent Robots and Systems, 2009.

[36] D. A. Winter, "Biomechanics and motor control of human movement," John Wiley \& Sons, 2009.

[37] F. Zhang et al., "Effects of locomotion mode recognition errors on volitional control of powered above-knee prostheses." IEEE Trans. Neur. Sys. Reh., vol. 23, no. 1, pp. 64-72, 2015.

[38] H. Huang et al., "Continuous locomotion-mode identification for prosthetic legs based on neuromuscular-mechanical fusion," IEEE Trans. Biomedical Engineering, vol. 58, no. 10, pp. 2867-2875, 2011.

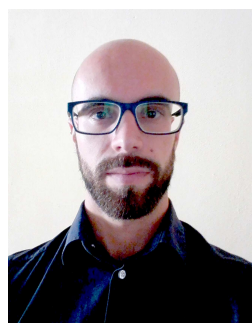

Andrea Parri received the M.Sc. degree in Biomedical Engineering (cum laude) from the University of Pisa, Italy, in April 2014. He is currently a Ph.D. student in Biorobotics at The BioRobotics Institue of Scuola Superiore SantAnna with the Wearable Robotics laboratory since November 2014. His major research interests are focused on the design and development of adaptive control architectures for lower-limb assistive wearable robots and their validation with target end-users in in-lab and ecological settings.

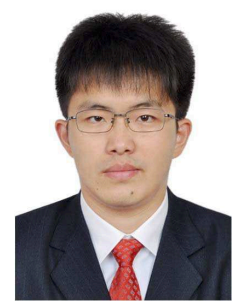

Kebin Yuan (S13) received the Bachelor's degree in automation from Chongqing University, Chongqing, China, in 2010, and Ph.D. degree in the Intelligent Control Laboratory, College of Engineering, Peking University, Beijing, China in 2016. He is now a senior engineer of Huawei Technology. His research interests include locomotion mode recognition and motion control of robotic rehabilitation devices.

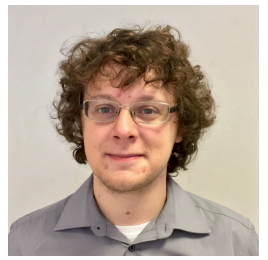

Dario Marconi received the M.Sc degree in biomedical engineering from the University of Pisa, Pisa, Italy, in 2015. He is currently a Ph.D. student in Biorobotics at The BioRobotics Institute, Scuola Superiore SantAnna, within the Wearable Robotics Laboratory. His research activities include the development of control strategies for robot-assisted hand rehabilitation and the assessment of functional requirement for hand exoskeletons.

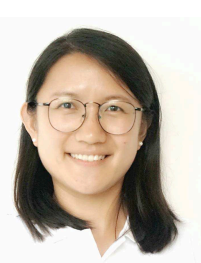

Tingfang Yan received her M.Sc. degrees in Control Science and Engineering from Shandong University, China, in 2014. In 2016, she received the $\mathrm{PhD}$ degree (cum laude) in The BioRobotics Institute, Scuola Superiore SantAnna, Pisa, Italy. Currently, she is a Research Associate in the Department of Electronic Engineering, The Chinese University of Hong Kong, Hong Kong, China. Her main research interests include designing assistive and rehabilitation exoskeleton systems and developing assistive strategies for wearable robotics.

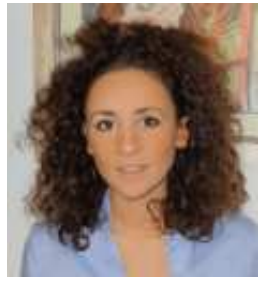

Simona Crea (M17) received her $\mathrm{PhD}$ in Biorobotics from Scuola Superiore Sant'Anna on December 2015. Since April 2017 she is Assistant Professor at The BioRobotics Institute of Scuola Superiore Sant'Anna, where she works on wearable robotics. Her research activities are focused on developing and validating novel wearable technologies and paradigms of human-robot interaction, with a strong focus on ergonomics and behavioral aspects. She is project manager of the H2020 CYBERLEGs Plus Plus project, and co-PI of two national projects funded by INAIL (the National Institute for Insurance against Accidents at Work), namely the MOTU and Habilis projects, and co-PI of the CENTAURO project funded by Regione Toscana.

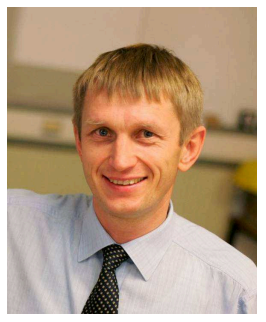

Marko Munih (M88) received the Ph.D. degree in electrical engineering from the University of Ljubljana, Ljubljana, Slovenia in 1993. He is a Full Professor, Head of Robolab at University of Ljubljana. His early research interests were focused in functional electrical stimulation of paraplegic lower extremities with surface electrode systems, including measurements, control, biomechanics and electrical circuits. Past research included robots in interaction with environment, design and use of haptic interfaces in rehabilitation. The industrial expertise covered robots construction and dedicated robot problems in the industry. Great interest was for contactless measurements of kinematics using Inertial Measurement Units (IMU). He (co)authored 120 reviewed journals publications, 5 patents and 4 textbooks. 


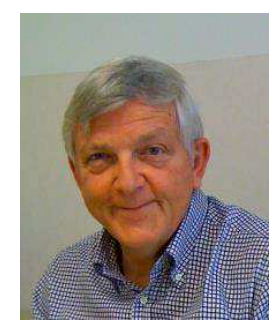

Raffaele Molino Lova is a Cardiologist and Cardiovascular Surgeon and has been involved in the clinical management of the Cardiac Rehabilitation Unit of Fondazione Don Gnocchi, Centre of Florence. At the moment he is Senior Researcher and his research interest is in the field of exercise physiology in older persons, in gas exchange kinetics and in the energy cost of walking. He is co-author of 75 ISI peerreviewed papers and he has been acknowledged by the Italian Ministry of Education as Full Professor in Rehabilitation Medicine on a national basis. He was PI in the EU FP7 CYBERLEGs project and he is PI of the the H2020-ICTCYBERLEGs Plus Plus project and of the national project MOTU funded by INAIL. He is co-coordinator of the Movement Assistance and Rehabilitation Laboratory, MARe Lab at Fondazione Don Gnocchi, Florence.

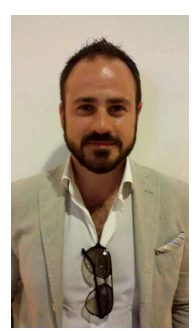

Nicola Vitiello (M12) received the MSc degree in biomedical engineering from the University of Pisa, Italy, in 2006, and from Scuola Superiore SantAnna (SSSA), Pisa, Italy, in 2007. He also received the $\mathrm{PhD}$ degree in Biorobotics from SSSA, in 2010. He is currently Associate Professor with The BioRobotics Institute, SSSA where he leads the Wearable Robotics Laboratory. He is the author or co-author of more than $50 \mathrm{ISI} / \mathrm{Scopus}$ papers and more than 30 peer-review conference proceedings papers and 17 patent applications. He has served as the Scientific Secretary of the EU FP7 CA-RoboCom project, and he was the scientific project coordinator of the EU FP7 CYBERLEGs project, the EARLYREHAB project funded by Regione Toscana, and the IUVO research project funded by a local bank Foundation, namely Fondazione Pisa. Currently he is the scientific project coordinator of the H2020-ICT-CYBERLEGs Plus Plus project, the national project MOTU funded by INAIL, and is partner of the H2020-ICT-AIDE and H2020-FoF-HUMAN projects. His interests include the development, experimental validation and maturation of novel wearable robotic devices for human movement assistance, rehabilitation and augmentation. He is a co-founder and shareholder of the spin-off company IUVO S.r.1.

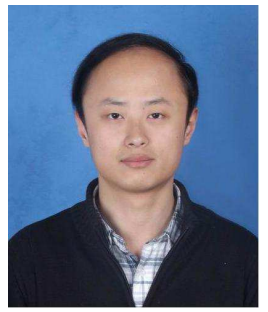

Qining Wang (S06 - M09) received the Ph.D. degree in dynamics and control from Peking University, Beijing, China, in 2009. He is currently a Tenured Associate Professor in the College of Engineering, Peking University, Co-PI of the Beijing Innovation Center for Engineering Science and Advanced Technology, and the Director of the Beijing Engineering Research Center of Intelligent Rehabilitation Engineering. He is the Project Leader of the wearable robotics R\&D Group. He has authored/coauthored over 130 scientific papers in international journals and refereed conference proceedings. His research interests include bio-inspired robots and rehabilitation robotics. Dr. Wang received the Best Technical Paper Award at the 17th International Conference on Climbing and Walking Robots (CLAWAR) in 2015 and the 18th International Conference on CLAWAR in 2016. He serves as an Advisor of the IEEERAS Technical Committee on Wearable Robotics, an Associate Editor for the IEEE Robotics and Automation Magazine and a Technical Editor for the IEEE/ASME Transactions on Mechatronics. 